\title{
Optical Recording of Action Potentials with Second-Harmonic Generation Microscopy
}

\author{
Daniel A. Dombeck, ${ }^{1}$ Mireille Blanchard-Desce, ${ }^{2}$ and Watt W. Webb ${ }^{1}$ \\ ${ }^{1}$ School of Applied and Engineering Physics, Cornell University, Ithaca, New York 14853, and ${ }^{2}$ Synthese et Electrosynthese Organiques (Unité Mixte de \\ Recherche 6510, Centre National de la Recherche Scientifique), Institut de Chimie, Universite de Rennes 1, F-35042 Rennes, France
}

\begin{abstract}
Nonlinear microscopy has proven to be essential for neuroscience investigations of thick tissue preparations. However, the optical recording of fast $(\sim 1 \mathrm{msec})$ cellular electrical activity has never until now been successfully combined with this imaging modality. Through the use of second-harmonic generation microscopy of primary Aplysia neurons in culture labeled with 4-[4(dihexylamino)phenyl][ethynyl]-1-(4-sulfobutyl)pyridinium (inner salt), we optically recorded action potentials with $0.833 \mathrm{msec}$ temporal and $0.6 \mu \mathrm{m}$ spatial resolution on soma and neurite membranes. Second-harmonic generation response as a function of change in membrane potential was found to be linear with a signal change of $\sim 6 \% / 100 \mathrm{mV}$. The signal-to-noise ratio was $\sim 1$ for single-trace action potential recordings but was readily increased to $\sim 6-7$ with temporal averaging of $\sim 50$ scans. Photodamage was determined to be negligible by observing action potential characteristics, cellular resting potential, and gross cellular morphology during and after laser illumination. High-resolution (micrometer scale) optical recording of membrane potential activity by previous techniques has been limited to imaging depths an order of magnitude less than nonlinear methods. Because second-harmonic generation is capable of imaging up to $\sim 400 \mu \mathrm{m}$ deep into intact tissue with submicron resolution and little out-of-focus photodamage or bleaching, its ability to record fast electrical activity should prove valuable to future electrophysiology studies.
\end{abstract}

Key words: imaging; action potential; membrane potential; second-harmonic generation; microscopy; nonlinear; optical; voltagesensitive dye

\section{Introduction}

The investigation of the electrical signaling properties of excitable cells such as neurons is predominately accomplished through the use of intracellular microelectrodes. Although these studies are useful for obtaining temporal electrical activity from a few locations on a single neuron (Stuart and Sakmann, 1994), they reveal little about the spatiotemporal modulations of membrane potential $\left(V_{\mathrm{m}}\right)$ over the entire cell or the activity of a population of neurons. Optical methods for monitoring $V_{\mathrm{m}}$ enable a greater understanding of the mechanisms underlying single neuron firing properties and cooperative electrical signaling in groups of neurons.

It is currently possible to follow cellular $V_{\mathrm{m}}$ activity optically through the use of linear one-photon methods (i.e., fluorescence, absorption, scattering, and birefringence). Slow transmembrane redistribution of dyes allows for $V_{\mathrm{m}}$ imaging with a high signalto-noise ratio $(\mathrm{S} / \mathrm{N})$ but cannot provide the $1 \mathrm{msec}$ temporal

Received 0ct. 28, 2003; revised Dec. 1, 2003; accepted Dec. 1, 2003.

This work was supported by Centre National de la Recherche Scientifique (Action Coordonnée Optique and Physique et Chimie du Vivant grants) to M.B.D. and National Institutes of Health Grants 9 P41 EB001976-16 and GM08267 to D.A.D. and W.W.W. We thank T. Mallegol for the synthesis of the DHPESBP dye and J. Mertz, B. Johnson, P. Kloppenburg, and many Webb group members for helpful discussions and careful reading of this manuscript. We appreciate the R. Harris-Warrick laboratory for the use of its microelectrode puller and for electrophysiology advice and guidance.

Correspondence should be addressed to Watt W. Webb, Applied Physics, Cornell University, 223 Clark Hall, Ithaca, NY 14853. E-mail: www2@cornell.edu.

DOI:10.1523/JNEUROSCI.4840-03.2004

Copyright $\odot 2004$ Society for Neuroscience $\quad$ 0270-6474/04/240999-05\$15.00/0 resolution needed to record fast $V_{\mathrm{m}}$ signals (Rink et al., 1980). Elegant green fluorescent protein (GFP) constructs (Knopfel et al., 2003) and fluorescence resonance energy transfer pairs (Gonzalez and Tsien, 1997) have been used to record $V_{\mathrm{m}}$ but can be limited either in their response time or in their ability to stain intact tissue (Zochowski et al., 2000), respectively. Methods using intrinsic changes in linear scattering or birefringence have been used to record action potentials (APs) in thin specimens (Cohen et al., 1968; Stepnoski et al., 1991), but recent attention has focused on fluorescent probes. These fast probes can respond to a $V_{\mathrm{m}}$ change of $100 \mathrm{mV}$ with up to $10-20 \%$ changes in fluorescence emission (Grinvald et al., 1982; Loew et al., 1992; Rohr and Salzberg, 1994), although the response is typically limited to $~ 1 \%$ in practice (Zochowski et al., 2000).

An innovative combination of high dye concentration, large illumination intensities, large collection areas, and/or very sensitive light detectors has allowed researchers to overcome these small signal changes and to image APs with an $\mathrm{S} / \mathrm{N}$ of $\sim 10$ and subthreshold events by temporal averaging at a spatiotemporal resolution of $\sim 10 \mu \mathrm{m}$ and $<1 \mathrm{msec}$ (Zochowski et al., 2000). However, in thick preparations, high-resolution (micrometer scale) one-photon techniques are limited to imaging depths of approximately $<50 \mu \mathrm{m}$ by light scattering, making the poor spatial resolution deep in scattering tissues (such as neural tissue) the most severe limitation.

Nonlinear microscopy [such as two-photon fluorescence, second-harmonic $(\mathrm{SH})$ generation $(\mathrm{SHG})$, etc.] has the potential 
to solve this problem because of its ability to image at depths of up to $\sim 400 \mu \mathrm{m}$ in scattering tissues with submicron resolution (Denk et al., 1990; Denk and Svoboda, 1997; Dombeck et al., 2003). Cells labeled with asymmetric styryl dyes generate an SH signal that is sensitive to $V_{\mathrm{m}}$ (Bouevitch et al., 1993; Ben-Oren et al., 1996; Campagnola et al., 1999). These dyes label only the outer leaflet of the lipid bilayer and therefore satisfy the noninversion symmetry requirement for the generation of an $\mathrm{SH}$ signal. Like other forms of nonlinear microscopy, SHG microscopy has imaging-resolution advantages over one-photon techniques deep in tissue and therefore has been the focus of considerable research to prove its capability to record fast $(\sim 1 \mathrm{msec})$ cellular $V_{\mathrm{m}}$ signals such as APs. However, its sensitivity to $V_{\mathrm{m}}$ has only been demonstrated on fast time scales in model membrane systems such as giant unilamellar vesicles (Moreaux et al., 2003; Pons et al., 2003) or on slow time scales ( $\sim 1 \mathrm{sec})$ in sustained cultured cell lines (Milliard et al., 2003). To date, there has been no demonstration of the ability to record fast $V_{\mathrm{m}}$ activity in living cells with any form of nonlinear microscopy, and therefore $\sim 1$ msec, high spatial resolution optical $V_{\mathrm{m}}$ recording has been limited to thin preparations or superficial regions of thick specimens. Here, we report the response of SHG from labeled Aplysia cell membranes during step changes in $V_{\mathrm{m}}$ and demonstrate $0.833 \mathrm{msec}$ and $0.6 \mu \mathrm{m}$ spatiotemporal resolution of APs with minimal background and photodamage during recording sessions.

\section{Materials and Methods}

Primary cell culture. Abdominal and cerebral ganglia from up to 15 different 5-10 gm Aplysia californica were dissociated and cultured with like ganglia (i.e., abdominal with abdominal, cerebral with cerebral) according to Goldberg and Schacher (1998). The cultures were grown in glassbottom dishes (MatTek, Ashland, MA) at $17-18^{\circ} \mathrm{C}$. For recordings, 4 - to 14-d-old cultures were used.

Staining and imaging. We used 4-[4-(dihexylamino)phenyl] [ethynyl]1-(4-sulfobutyl)pyridinium (DHPESBP) (inner salt) [molecule B from Moreaux et al. (2003)] because of its proven fast $(<150 \mu \mathrm{sec})$ response to $V_{\mathrm{m}}$. SHG from giant unilamellar vesicles stained with this molecule was sensitive to $V_{\mathrm{m}}$ on fast time scales through an electrochromic (rather than reorientational) mechanism (Moreaux et al., 2003). Here, staining was accomplished by extracellular perfusion of $8 \mu \mathrm{M}$ dye in SL-15 extracellular buffer (Goldberg and Schacher, 1998). The dye remained in the bath during the experiments, and the flip-flop time of a similar dye to the inner leaflet of a stained membrane was found previously to be $\sim 2 \mathrm{hr}$ (Moreaux et al., 2001). The SHG imaging system has been described previously (Dombeck et al., 2003), except here, the transmitted lightcondensing objective was replaced by a 0.9 numerical aperture (NA) condenser, modified to switch easily between differential interference contrast wide-field imaging and SHG collection. The focusing objective was also changed to a $20 \times, 0.75$ NA Zeiss (Thornwood, NY) Fluar, making a diffraction-limited $\sim 0.6 \mu \mathrm{m}$ radial diameter focal spot. The Ti:Sapphire laser was operated at $940 \mathrm{~nm}$ with $\sim 12 \mathrm{~mW}$ of average power at the sample, and a 460/30 bandpass filter was used for SHG detection in front of the bialkali photomultiplier tube. The excitation polarization was linear.

Optical and electrode voltage recording. Electrophysiological recordings of Aplysia neuron cultures were made at room temperature with an Axoclamp 2B amplifier and pClamp 8.1 software (Axon Instruments, Union City, CA). Two-electrode voltage clamp was used to clamp $V_{\mathrm{m}}$ at defined values. For stimulation of APs, the neuron was impaled with two microelectrodes (8-15 M $\Omega$, filled with $3 \mathrm{M} \mathrm{KCl}$ ), one for voltage recording and the other for current injection. AP stimulation was possible with $<5 \mathrm{nA}, \sim 50 \mathrm{msec}$ duration current pulses as used previously (Bedi et al., 1998; Rubakhin et al., 1999); however, shorter more intense pulses (30$100 \mathrm{nA}, 1.0-3.5 \mathrm{msec}$ duration) were used here to quickly reach threshold. This shorter stimulation protocol afforded an AP temporal stability of $<0.5 \mathrm{msec}$ peak voltage drift over the minutes of signal averaging that was not possible with the longer protocol ( $>3 \mathrm{msec}$ drift), obviating the need for postprocessing. The necessary temporal resolution to optically detect APs with SHG was obtained using the line-scanning mode of the scanning system (600-1200 lines/sec). X-Y images were taken by raster scanning the focal spot over the two-dimensional field of view while one line was repeatedly scanned 256 times during line scanning. Each 8-bit image is built of 256 pixels in each dimension. The line scanning and stimulation or voltage-clamping steps were synchronized through trigger pulses coupling the amplifier and image acquisition software. Exact timing of the amplifier signals and line scanning was accomplished by recording the monitor output of the amplifier in one of the line-scan imaging channels. This enabled an increase in the $\mathrm{S} / \mathrm{N}$ through line-scan averaging. Each recording session consisted of a number $(n)$ of stimulation-line scans in series with pauses, $4 \mathrm{sec}$ for two-electrode voltage clamp and $3 \mathrm{sec}$ for AP stimulation experiments, to save the images and electrode recordings and reset the system for the next line scan. A typical $n=50$ session totaled $2-3 \mathrm{~min}$ in duration.

Dye synthesis. The electroNLOchromic dye DHPESBP was obtained via a multistep synthesis scheme involving a Sonogashira key coupling reaction followed by alkylation with 1,4 butane sultone (T. Mallegol, $\mathrm{O}$. Mongin, M. Blanchard-Desce, unpublished procedure).

\section{Results}

\section{Spatiotemporal AP recordings with SHG microscopy}

Figure 1, $A$ and $B$, shows SHG images of a cultured Aplysia neuron stained with DHPESBP. Because of the noninversion symmetry requirement of SHG, a signal is only produced at the labeled membranes; no background from randomly oriented dye is seen. During line scanning of the green line in Figure $1 B$, voltage steps were applied to the cell in voltage clamp. The $\mathrm{S} / \mathrm{N}$ was $\sim 1$ for single traces, necessitating temporal averaging. This was accomplished by synchronizing the start of the line scan with the amplifier voltage steps. After repeated line-scan averaging $(n=$ 50 ), the modulations in the SHG emission followed the voltage steps accurately with an $\mathrm{S} / \mathrm{N}$ of $\sim 7$. This line-scan average is scaled to the dynamic range of the SHG response to visualize the small change in emission (Fig. 1C). Negative voltage steps resulted in increased SHG emission, whereas positive voltage steps resulted in decreased emission (Fig. $1 D$ ). A linear response of the SHG with respect to $\Delta V_{\mathrm{m}}$ (linear best fit: $\Delta \mathrm{SHG} / \mathrm{SHG}=$ $-0.06 \Delta V_{\mathrm{m}}$; slope error $\left.=0.004\right)$ was observed by applying a range of voltage steps (Fig. $1 E$ ).

To prove the ability of SHG to record fast neuronal signals, APs were elicited and optically recorded. Line-scanning positions of two different recording sessions on an Aplysia neuron are represented by the green lines in Figure $2 A$, one through the soma and the other through several neurites. During each line scan, two $\sim 10$ - to 15 -msec-duration APs, consistent with mixed $\mathrm{Ca}^{2+}$ $\mathrm{Na}^{+}$APs (Gardner and Kerkut, 1968), were elicited through intracellular electrodes (Fig. $2 B, C$, red traces). Line-scan averaging $(n=50)$ showed that the optical intensity modulations in the SHG emission follow the fast $V_{\mathrm{m}}$ events with $0.6 \mu \mathrm{m}$ spatial and $0.833 \mathrm{msec}$ temporal resolution and an $\mathrm{S} / \mathrm{N}$ of $\sim 6$ (Fig. $2 B, C$, green traces). Note that the somatic recording electrode and SHG optical recording positions are different, so the AP shape and duration differ in some instances, as seen previously (Zecevic, 1996). The temporal response of SHG is easily capable of recording faster events such as the $\sim 4$ msec duration APs, consistent with $\mathrm{Na}^{+}$APs (Gardner and Kerkut, 1968), seen in Figure $2 D$. After line-scan averaging $(n=70)$, the $\mathrm{S} / \mathrm{N}$ was $\sim 7$ for this recording session.

The $\mathrm{S} / \mathrm{N}$ in SHG recording can be understood by analyzing the noise in the baseline of the optical traces. For example, noise of $\sim 1 \%$ is seen in the baseline of Figure 2, $B$ and $C$. Assuming a shot noise-limited system, this equates to a total of $\sim 10,000$ photons collected per membrane during the 50 traces, yielding $\sim 200$ pho- 

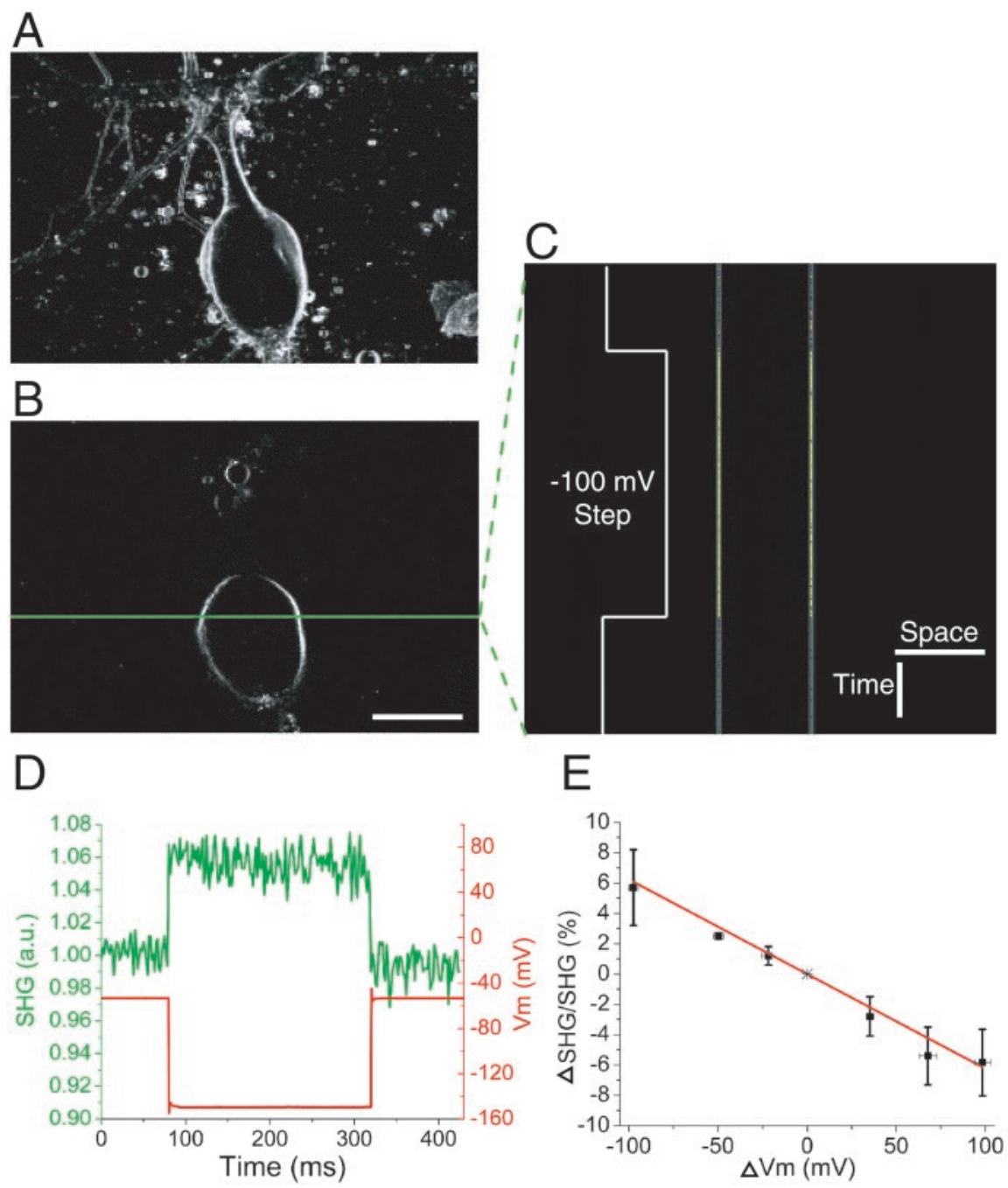

Figure 1. Line-scan recording of $V_{m}$ with SHG during voltage steps in cultured Aplysia neurons. $A$, Projection image superimposing $21 \sim 1$ - $\mu \mathrm{m}$-thick z-sections $2 \mu \mathrm{m}$ apart. $B$, A single z-section through the neuron in $A$ at the plane of line scanning. The green line represents the scanned line where membrane potentials are recorded. C, SHG signal changes recorded by line scanning the line denoted in $B$ at 600 lines/sec. The voltage-clamped neuron was given a $240 \mathrm{msec}$ duration $-100 \mathrm{mV}$ step after $80 \mathrm{msec}$ of scanning during each line scan. $n=50$ line scans were averaged. The line scan image is scaled to visualize the small change in SHG emission. Calibration: $50 \mu \mathrm{m}, 50 \mathrm{msec}$. D, The green trace, obtained from the left membrane line in $C$, is a normalized intensity plot of SHG emission versus time. The red line represents the measured $V_{m}$ during the voltage-clamp step. a.u., Arbitrary units. $E$, Plot of $\Delta S H G / S H G$ over physiologically relevant $\Delta V_{\mathrm{m}}$. The functional fit in red shows a linear relationship. Error bars represent SDs of three to five different measurements at each $\Delta V_{\mathrm{m}} \pm \sim 4 \mathrm{mV}$. Note the inverse relationship between $\Delta S H G / S H G$ and $\Delta V_{\mathrm{m}}$

tons during the laser dwell time across the membrane. Therefore, the noise is $\sim 7 \%$ and the $\mathrm{S} / \mathrm{N}$ is $\sim 1$ for $n=1$.

\section{Minimal phototoxicity during recordings}

Little cellular photodamage was detected during the recording sessions with an average power of $\sim 12 \mathrm{~mW}$, as in those examples presented here. To evaluate this, we first noted that the variation in resting potential was $<4 \mathrm{mV}$ during individual sessions, equivalent to nonillumination controls. Second, the elicited APs remained constant in amplitude and duration throughout recording sessions compared with nonillumination controls. Finally, no gross morphological changes were detectable in the soma or processes after individual sessions. Repeated optical recording from single neurons was possible over the course of $\sim 0.5 \mathrm{hr}$. When the power was increased to $\sim 22 \mathrm{~mW}$, damage was observable. Recording from the soma was still possible with little damage, but recording from distal neurites resulted in blebbing or transection of the processes at the sites of line scanning. More studies are needed to investigate long-term damage effects and to determine the cause of the phototoxicity and possible avoidance procedures. Although little loss of the SHG signal was observed at $\sim 12 \mathrm{~mW}$, it was more apparent at $\sim 22 \mathrm{~mW}$.

\section{Discussion}

An eventual goal of optical $V_{\mathrm{m}}$ recording is to image subthreshold events, without averaging, deep in intact neural tissue with $<1 \mu \mathrm{m}$ and $\sim 1$ msec spatiotemporal resolution. To reach this goal, the SHG S/N for $V_{\mathrm{m}}$ recording and imaging frame rate must be improved (i.e., full twodimensional images must be obtained at the same temporal resolution demonstrated here for line scanning). Commonly used commercial scanning systems have a frame rate for full images of $\sim 1 \mathrm{~Hz}$; thus, to obtain the necessary $1 \mathrm{kHz}$ imaging frame rate, new scanning technologies must be implemented. Resonant galvanometer (Tsien and Bacskai, 1995)- and microlens (Kobayashi et al., 2002)-based scanners have been used for this purpose, but current demonstrations of these devices only achieve a frame rate of $\sim 33 \mathrm{~Hz}$. Newer versions of this technology may show promise for faster scan rates (So, 2003). Another possibility is the use of random access scanning (Bullen et al., 1997; Bullen and Saggau, 1999) to scan over only the membrane region of interest.

Improvements in $\mathrm{S} / \mathrm{N}$ can come from: (1) increased laser power, (2) higher dye concentrations in the membrane, (3) higher quantum yield detectors, or (4) better SHG molecules, with increases in both hyperpolarizibility and response to $V_{\mathrm{m}}$. The number of emitted photons is proportional to the square of both the dye concentration and the fundamental laser intensity (Moreaux et al., 2000). Therefore, increases in either should lead to large decreases in the photon shot noise; methods for decreasing photodamage must be investigated concurrently. Rapid progress is being made on option 3. New GaAsP photomultiplier tubes, for example, have greater quantum efficiency than the bialkali model used here. Option 4 will involve the design and screening of many dyes. It is likely that molecules with greater $V_{\mathrm{m}}$ response and increased hyperpolarizibility will be found.

To achieve the goal of $V_{\mathrm{m}}$ imaging with SHG under the guidelines above, many possible variables may be altered. For example, an $\sim 20 \% \Delta$ SHG/100 $\mathrm{mV}$ dye with twice the hyperpolarizibility of DHPESBP, stained and illuminated at twice the concentration and incident power used here, should be capable of recording a 10 $\mathrm{mV}$ subthreshold event with $n=1$ and an $\mathrm{S} / \mathrm{N}$ of $>1$.

Recording $V_{\mathrm{m}}$ activity deep in tissue may be possible in the near future, but sample thickness, staining procedures, and tissue 

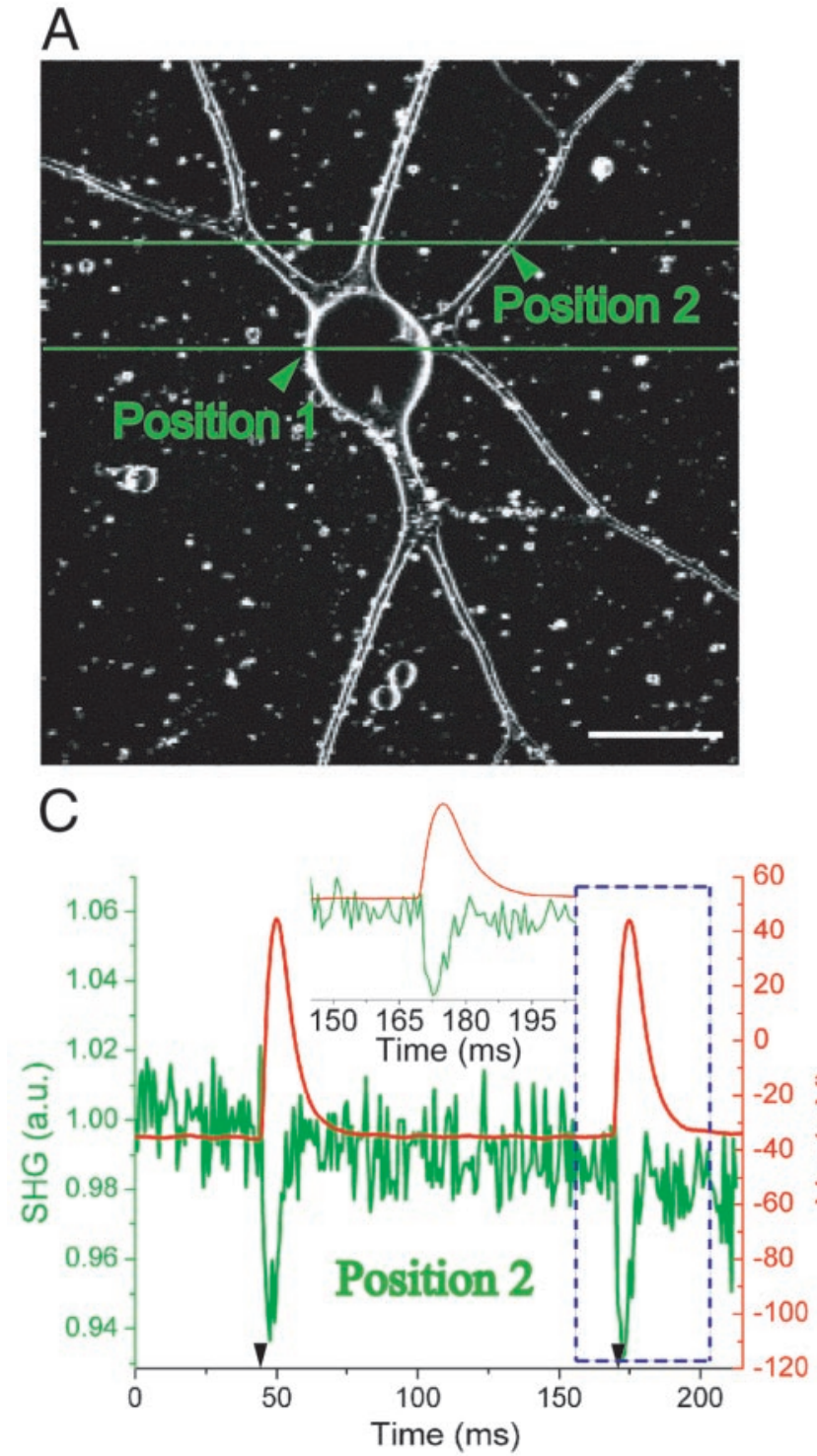
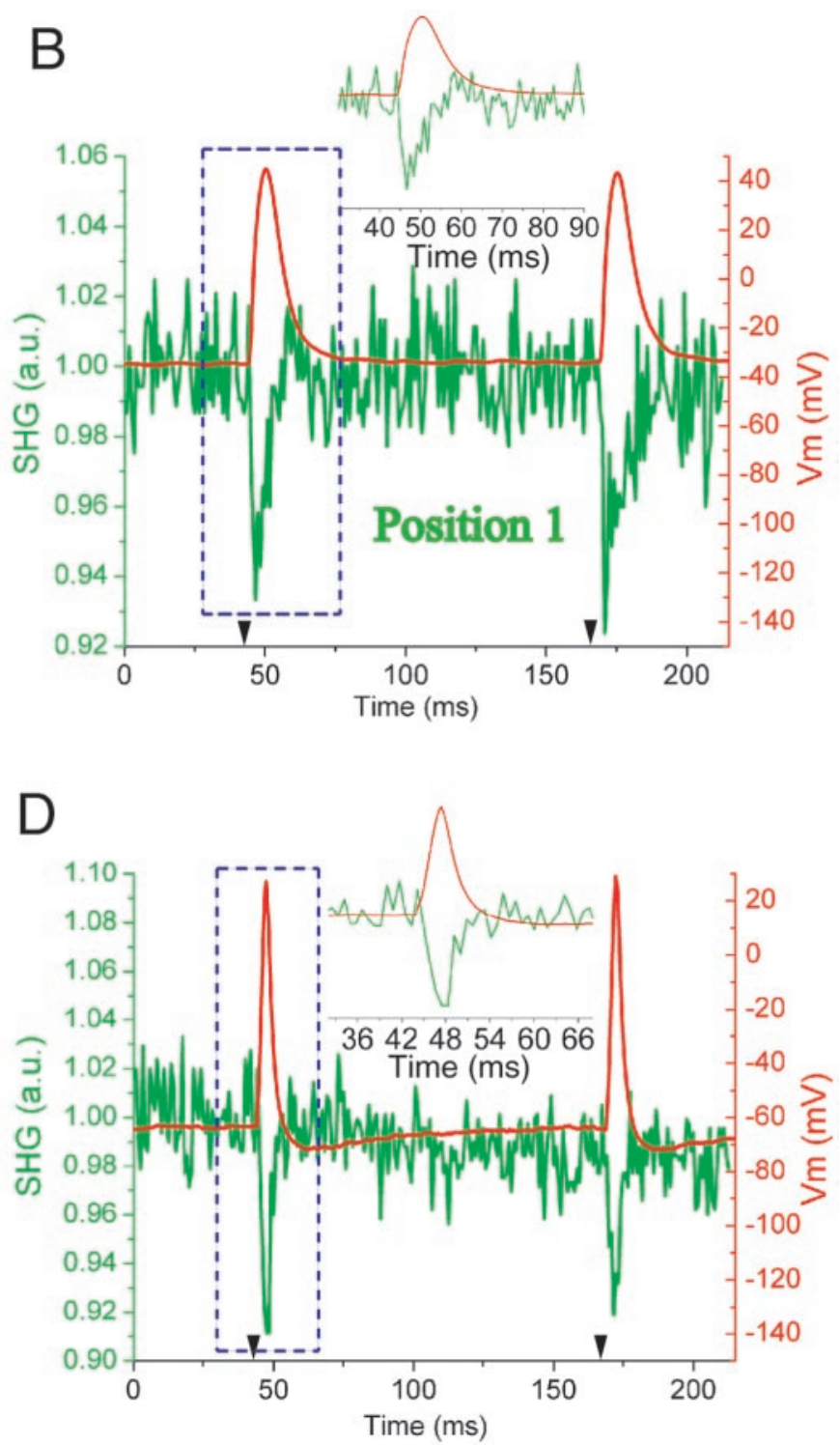

Figure 2. Fast SHG line-scan recording of APs at multiple sites in cultured Aplysia neurons. $A$, An SHG projection image of six $\sim 1$ - $\mu$ m-thick z-sections $3 \mu \mathrm{m}$ apart. Green lines represent the scanned lines. Scale bar, $50 \mu \mathrm{m}$. B, C, The green traces, obtained from the averaged line scans, are normalized intensity plots of SHG emission versus time at membrane position 1 and position 2 , respectively, in $A$ at 1200 lines/sec ( $0.833 \mathrm{msec} /$ line). Two APs were elicited by current injection (timing of current pulses given by black arrowheads), one at $45 \mathrm{msec}$ and the other at $170 \mathrm{msec}$, during each line scan. $n=50$ line scans were averaged. The red trace is the $V_{\mathrm{m}}$ from the recording electrode at the soma. The inset is an expanded time base around the AP in the blue dashed box. C reveals the usefulness of high-resolution optical $V_{\mathrm{m}}$ recording by showing the clear difference in AP duration that can occur between the soma and neurites of individual neurons. $D$, Example of shorter APs from a different neuron than in $A . n=70$ line scans were averaged.

type must first be carefully evaluated. Intrinsic SHG imaging at depths of up to $300-400 \mu \mathrm{m}$ with submicron resolution has been demonstrated previously in hippocampal brain slices (Dombeck et al., 2003). Because SHG is collected in the transmitted light direction, sample thickness is limited to approximately $<500$ $\mu \mathrm{m}$. Staining thick turbid media, such as intact ganglia or mammalian neural tissue, has been accomplished by bath-applying dye (Delaney et al., 1994), but other techniques such as pressure injection of dye (Stosiek et al., 2003), intracellular labeling (Antic et al., 1999), novel GFP constructs (Knopfel et al., 2003), or the addition of dye crystals into tissue (Gan et al., 2000; Moreaux et al., 2001) have been needed previously to stain at these depths. Additionally, varying preparations have led previously to different sensitivities of the same $V_{\mathrm{m}}$ dyes (Zochowski et al., 2000), but a greater issue for SHG dyes may be their effects on more delicate mammalian cells.
With these issues in mind, numerous applications should be possible with SHG $V_{\mathrm{m}}$ recording. The current capabilities demonstrated here are most immediately applicable to reproducibly stimulated systems, as are often used for current optical $V_{\mathrm{m}}$ recording techniques (Zecevic, 1996). It should be feasible to investigate spike initiation zones (Zecevic, 1996) and AP propagation properties (Fromherz and Muller, 1994; Meyer et al., 1997) deep in intact ganglia, where one-photon methods are not appropriate. Repeated line scans at varying spatial positions over processes will allow for the generation of high-resolution time series movies of AP propagation, leading to the experimental analysis of electrical propagation properties at complex structures, such as axon bifurcations and varicosities. In addition, SHG $V_{\mathrm{m}}$ recording simultaneously with powerful $\mathrm{Ca}^{2+}$ imaging techniques (Szmacinski et al., 1993; Yuste and Denk, 1995) should prove to be a useful combination. 
Any increase in spatial resolution is valuable for the investigation of $V_{\mathrm{m}}$ properties of small structures (i.e., dendritic spines) or for providing higher-resolution data of AP propagation. The spatial resolution of $\sim 0.6 \mu \mathrm{m}$ demonstrated here represents the highest-resolution optical recording of fast $V_{\mathrm{m}}$ activity to date. The potential usefulness of this resolution for AP propagation studies in which shape and duration vary depending on position is seen in Figure 2C, where these differences are clear. Theoretically, similar spatial resolution is possible for the fast one-photon techniques while maintaining the same $\mathrm{S} / \mathrm{N}$; however, a higher illumination intensity would be needed, likely resulting in greater photodamage. The highest demonstrated resolution of $\sim 2 \mu \mathrm{m}$ was demonstrated by one-photon random-access scanning, but like other linear methods, it was limited to thin specimens (Bullen et al., 1997; Bullen and Saggau, 1999).

Here, we demonstrated that SHG microscopy is capable of recording APs from excitable neurons with $0.833 \mathrm{msec}$ temporal resolution at the highest spatial resolution yet reported for fast optical $V_{\mathrm{m}}$ recordings. This technique for optically recording $V_{\mathrm{m}}$ holds the following important advantages over current techniques: submicron resolution at depths up to $\sim 400 \mu \mathrm{m}$ in scattering tissue, little out-of-focus photodamage or bleaching, and no background. The above work represents a key step toward $\sim 1$ msec time-scale, high-resolution $V_{\mathrm{m}}$ imaging deep in intact neural tissue. We expect that rapid progress will continue to be made toward this goal in the near future.

\section{References}

Antic S, Major G, Zecevic D (1999) Fast optical recordings of membrane potential changes from dendrites of pyramidal neurons. J Neurophysiol 82:1615-1621.

Bedi SS, Salim A, Chen S, Glanzman DL (1998) Long-term effects of axotomy on excitability and growth of isolated Aplysia sensory neurons in cell culture: potential role of cAMP. J Neurophysiol 79:1371-1383.

Ben-Oren I, Peleg G, Lewis A, Minke B, Loew L (1996) Infrared nonlinear optical measurements of membrane potential in photoreceptor cells. Biophys J 71:1616-1620.

Bouevitch O, Lewis A, Pinevsky I, Wuskell JP, Loew LM (1993) Probing membrane potential with nonlinear optics. Biophys J 65:672-679.

Bullen A, Saggau P (1999) High-speed, random-access fluorescence microscopy. II. Fast quantitative measurements with voltage-sensitive dyes. Biophys J 76:2272-2287.

Bullen A, Patel SS, Saggau P (1997) High-speed, random-access fluorescence microscopy. I. High-resolution optical recording with voltagesensitive dyes and ion indicators. Biophys J 73:477-491.

Campagnola PJ, Wei MD, Lewis A, Loew LM (1999) High-resolution nonlinear optical imaging of live cells by second harmonic generation. Biophys J 77:3341-3349.

Cohen LB, Keynes RD, Hille B (1968) Light scattering and birefringence changes during nerve activity. Nature 218:438-441.

Delaney KR, Gelperin A, Fee MS, Flores JA, Gervais R, Tank DW, Kleinfeld D (1994) Waves and stimulus-modulated dynamics in an oscillating olfactory network. Proc Natl Acad Sci USA 91:669-673.

Denk W, Svoboda K (1997) Photon upmanship: why multiphoton imaging is more than a gimmick. Neuron 18:351-357.

Denk W, Strickler JH, Webb WW (1990) Two-photon laser scanning fluorescence microscopy. Science 248:73-76.

Dombeck DA, Kasischke KA, Vishwasrao HD, Ingelsson M, Hyman BT, Webb WW (2003) Uniform polarity microtubule assemblies imaged in native brain tissue by second-harmonic generation microscopy. Proc Natl Acad Sci USA 100:7081-7086.

Fromherz P, Muller CO (1994) Cable properties of a straight neurite of a leech neuron probed by a voltage-sensitive dye. Proc Natl Acad Sci USA 91:4604-4608.

Gan WB, Grutzendler J, Wong WT, Wong RO, Lichtman JW (2000) Multicolor "DiOlistic" labeling of the nervous system using lipophilic dye combinations. Neuron 27:219-225.

Gardner DR, Kerkut GA (1968) A comparison of the effects of sodium and lithium ions on action potentials from Helix aspersa neurones. Comp Biochem Physiol 25:33-48.

Goldberg D, Schacher S (1998) Culturing large neurons of Aplysia. In: Culturing nerve cells, Ed 2 (Banker G, Goslin K, eds), pp 213-236. Cambridge, MA: MIT.

Gonzalez JE, Tsien RY (1997) Improved indicators of cell membrane potential that use fluorescence resonance energy transfer. Chem Biol 4:269-277.

Grinvald A, Hildesheim R, Farber IC, Anglister L (1982) Improved fluorescent probes for the measurement of rapid changes in membrane potential. Biophys J 39:301-308.

Knopfel T, Tomita K, Shimazaki R, Sakai R (2003) Optical recordings of membrane potential using genetically targeted voltage-sensitive fluorescent proteins. Methods 30:42-48.

Kobayashi M, Fujita K, Kaneko T, Takamatsu T, Nakamura O, Kawata S (2002) Second-harmonic generation microscope with a microlens array scanner. Opt Lett 27:1324-1326.

Loew LM, Cohen LB, Dix J, Fluhler EN, Montana V, Salama G, Wu JY (1992) A naphthyl analog of the aminostyryl pyridinium class of potentiometric membrane dyes shows consistent sensitivity in a variety of tissue, cell, and model membrane preparations. J Membr Biol 130:1-10.

Meyer E, Muller CO, Fromherz P (1997) Cable properties of dendrites in hippocampal neurons of the rat mapped by a voltage-sensitive dye. Eur J Neurosci 9:778-785.

Millard AC, Jin L, Lewis A, Loew LM (2003) Direct measurement of the voltage sensitivity of second-harmonic generation from a membrane dye in patch-clamped cells. Opt Lett 28:1221-1223.

Moreaux L, Sandre O, Mertz J (2000) Membrane imaging by secondharmonic generation microscopy. J Opt Soc Am B 17:1685-1694.

Moreaux L, Sandre O, Charpak S, Blanchard-Desce M, Mertz J (2001) Coherent scattering in multi-harmonic light microscopy. Biophys J 80:1568-1574.

Moreaux L, Pons T, Dambrin V, Blanchard-Desce M, Mertz J (2003) Electro-optic response of second-harmonic generation membrane potential sensors. Opt Lett 28:625-627.

Pons T, Moreaux L, Mongin O, Blanchard-Desce M, Mertz J (2003) Mechanisms of membrane potential sensing with second-harmonic generation microscopy. J Biomed Opt 8:428-431.

Rink TJ, Montecucco C, Hesketh TR, Tsien RY (1980) Lymphocyte membrane potential assessed with fluorescent probes. Biochim Biophys Acta 595:15-30.

Rohr S, Salzberg BM (1994) Multiple site optical recording of transmembrane voltage (MSORTV) in patterned growth heart cell cultures: assessing electrical behavior, with microsecond resolution, on a cellular and subcellular scale. Biophys J 67:1301-1315.

Rubakhin SS, Li L, Moroz TP, Sweedler JV (1999) Characterization of the Aplysia californica cerebral ganglion F cluster. J Neurophysiol 81:1251-1260.

So PTC (2003) Modern applications of 3-D optical microscopy in biology and medicine. Conference on Lasers and Electro-Optics-Quantum Electronics and Laser Science Conference, Baltimore, June.

Stepnoski RA, LaPorta A, Raccuia-Behling F, Blonder GE, Slusher RE, Kleinfeld D (1991) Noninvasive detection of changes in membrane potential in cultured neurons by light scattering. Proc Natl Acad Sci USA 88:9382-9386.

Stosiek C, Garaschuk O, Holthoff K, Konnerth A (2003) In vivo two-photon calcium imaging of neuronal networks. Proc Natl Acad Sci USA 100:7319-7324.

Stuart GJ, Sakmann B (1994) Active propagation of somatic action potentials into neocortical pyramidal cell dendrites. Nature 367:69-72.

Szmacinski H, Gryczynski I, Lakowicz JR (1993) Calcium-dependent fluorescence lifetimes of Indo-1 for one- and two-photon excitation of fluorescence. Photochem Photobiol 58:341-345.

Tsien RY, Bacskai BJ (1995) Video-rate confocal microscopy. In: Handbook of biological confocal microscopy, Ed 2 (Pawley JB, ed), pp 459-477. New York: Plenum.

Yuste R, Denk W (1995) Dendritic spines as basic functional units of neuronal integration. Nature 375:682-684.

Zecevic D (1996) Multiple spike-initiation zones in single neurons revealed by voltage-sensitive dyes. Nature 381:322-325.

Zochowski M, Wachowiak M, Falk CX, Cohen LB, Lam YW, Antic S, Zecevic D (2000) Imaging membrane potential with voltage-sensitive dyes. Biol Bull 198:1-21. 